\title{
Better outcomes in pulpotomies on primary molars with MTA
}

\author{
Is mineral trioxide aggregate more effective than formocresol for primary \\ molar pulpotomy?
}

\begin{abstract}
Peng L, Ye L, Tan $\mathbf{H}$, Zhou X. Evaluation of the formocresol versus mineral trioxide aggregate primary molar pulpotomy: a metaanalysis. Oral Surg Oral Med Oral Pathol Oral Radiol Endod 2006; 102:40-44
\end{abstract}

Data sources Medline, Cochrane Library, Embase, Science Citation Index and the China National Knowledge Infrastructure were used to source articles. In addition, identified papers' reference lists and their authors' other published literature were also scanned.

Study selection Studies of interest were randomised controlled trials of primary molar teeth where there was exposure of vital pulp caused by caries or trauma, with at least 6 months follow-up. It was required that selected teeth had no internal root resorption, inter-radicular and periapical bone destruction, no periodontal involvement, swelling, or sinus tract; they should be restorable with stainless steel crowns; and that outcomes were evaluated by clinical symptoms and radiographic evidence where the comparison followed a standard definition of success or failure, as shown by the number of teeth.

Data extraction and synthesis Data were extracted from each study independently and entered into a database. Differences were resolved by discussion. Studies were scored for validity criteria according to the Jadad scale. Meta-analysis was performed using the software RevMan (version 4.2.8; Copenhagen; The Nordic Cochrane Centre, The Cochrane Collaboration, 2003). Heterogeneity between studies was assessed using a standard chi-square test. If there was homogeneity among the studies, the fixed-effect model (Peto method) was applied to aggregate the data. If homogeneity was rejected then sensitivity analyses were performed using a random-effects model.

Results Six studies met the inclusion criteria (giving a total of 381 teeth). There was a significant difference between the success rates of formocresol (FC)- and mineral trioxide aggregate (MTA)-treated pulpotomised primary molars $(\mathrm{P}<0.05)$ Clinical assessments and radiographic findings of the MTA versus FC pulpotomy suggested that MTA was superior to $\mathrm{FC}$ in pulpotomy, resulting in a lower failure rate [relative risk, 0.32 (95\% confidence interval, $0.11-0.90$ ) and 0.31 (95\% confidence interval, 0.13-0.74) respectively].

Conclusions The results demonstrate that in primary molar teeth with vital pulp exposure caused by caries or trauma, a pulpotomy performed with MTA results in better clinically and radiographically observed outcomes. Fewer undesirable responses were recorded for MTA than when FC was used. Therefore, the study supports the use of MTA instead of FC as wound dressing when performing pulpotomies on primary molars.

Address for correspondence: Xuedong Zhou, Sichuan University, West China School of Dentistry, 3rd Section, South of Ren Ming Road, Chengdu, China. E-mail:pl_huaxi@sina.com

\section{Commentary}

This meta-analysis aimed to assess the clinical effectiveness of MTA versus $\mathrm{FC}$ in primary molar pulpotomy. Continuing concerns over the toxicological properties of formaldehyde as one of FC's main components have led to efforts to assess alternative materials for appropriateness and effectiveness. MTA is one such possibility.

This paper starts with an overview of the pulpotomy procedure, presentation of some of the evidence for FC's less desirable effects, including cytotoxicity and mutagenicity, and a short review of MTA with the emphasis on its biocompatibility. This is followed by justification for the use of meta-analysis as a tool for pooling data from individual studies to obtain an overall estimate for the effect of a treatment.

The methodology initially seems clear, presenting the search strategies and outcomes, unambiguous inclusion and exclusion criteria, treatment outcomes, and details of data extraction procedures. The analyses have checked for, and taken into account, heterogeneity between the studies.

There are a number of areas in this paper, however, that undermine confidence in the authors' conclusions. There is a Cochrane-type approach to the methods, and meta-analyses were carried out using the Cochrane Collaboration's software. The Jadad Scale was chosen, however, to assess the quality of the included papers. Two of the six papers only achieve a score of 2 (out of a possible 5), with no further details being provided. Also, of the six clinical trials identified from the search strategy and analysed (five of which were published after the Cochrane review on pulp treatments), ${ }^{1}$ one trial was included here yet excluded by the Cochrane review because the randomisation unit was the tooth and not the patient. Yet this was a requisite in this paper's inclusion criteria also.

There is also a lack of clarity in the followup periods. First, there is the observation period chosen, with the authors being unable to use a standard time: they chose the longest followup time available for each study, which is different for all but two of the studies. A sensitivity analysis might have indicated whether choosing different endpoints affected the results, although it is quite possible that the data reported in the individual trial papers would have been insufficient to allow extraction of this data. Second, the inclusion criteria state that the teeth should have been followed up for a minimum of 6 months, but two studies had shorter followup periods and there is no detail on how these data were handled.

Another area where a lack of detail compromises the paper's interpretation is where the inclusion criteria state that the teeth should be restorable with preformed metal crowns (PMC), but no data are presented as to how study teeth were restored. This is known to be a potential confounding factor in measuring the outcome of pulp treatment medicaments because teeth with PMC tend to fare better. ${ }^{2}$

Confidence in the results here is tempered by the lack of detail along with inconsistency between the stated inclusion/ exclusion 
criteria and the data presented. Having said that, the individual studies' data do seem to support the use of MTA as an alternative to FC in the vital pulpotomy procedure, although the degree of success that might be expected with MTA cannot be accurately quantified. The difficulty in interpreting the meta-analysis of the studies included here may have been avoided if the authors had followed the QUOROM guidelines: ${ }^{3}$ these would have improved reporting and thus interpretation of the results.

FC has been undergoing a process of rejection for several decades but there is still controversy over recommendations for its use. The recent Scottish Intercollegiate Guidelines Network guideline recommended that FC, "should be replaced by alternative materials for pulpotomy procedures in primary teeth" ${ }^{4}$ but the British Society of Paediatric Dentistry guidelines, ${ }^{5}$ when describing the procedure for a pulpotomy, suggest that one of the medicaments that can be chosen is a 1 in 5 dilution of Buckley's FC solution (although they do add that routine use of the FC pulpotomy "may be imprudent given the availability of effective alternatives").

This brings us back to the problem suffered by all attempts to evaluate clinical dental research: the low quality of the studies from which reviewers attempt to extract data. The authors discuss the poor randomisation methods: three studies used a coin toss and three failed to report how randomisation was achieved. Why do investigators going to such lengths as carrying out a clinical trial fail to follow accepted standards in methodology, such as using random number tables to generate randomisation, and then also do not report clearly what they did? The less rigorous randomisation is, the greater differences between control and interventions tend to be. ${ }^{5}$ The reason for the difficulty in changing recommendations is insecurity in the evidence base supporting the use of other medicaments such as MTA or calcium hydroxide (which is cheaper and more readily available than MTA), or ferric sulphate. Reaching this certainty requires meticulous implementation and reporting of clinical trials with standardised, comparable and patient-centred outcomes and this aim is something the paediatric dental community should embrace as a group.

\section{Practice Point}

From this paper, MTA does seem to be a reasonable medicament for use in pulpotomy procedures. Methodological weaknesses, however, mean that caution should be exercised in extrapolating to clinical practice the expected degree of success, from the data reported here.

\section{Nicola Innes}

Dental School, University of Dundee, Dundee, Scotland, UK

1. Nadin G, Goel BR, Yeung CA, Glenny AM. Pulp treatment for extensive decay in primary teeth. Cochrane Database Syst Rev 2003; issue 1.

2. Al-Zayer MA, Straffon LH, Feigal RJ, Welch KB. Indirect pulp treatment of primary posterior teeth: a retrospective study. Pediatr Dent 2003; 25:29-36.

3. Moher D, Cook DJ, Eastwood S, Olkin I, Rennie D, Stroup DF. Improving the quality of reports of meta-analyses of randomised controlled trials: the QUOROM statement. Quality of Reporting of Meta-analyses. Lancet 1999; 354:1896-1900.

4. Scottish Intercollegiate Guidelines Network. Prevention and management of dental decay in the pre-school child. A national clinical guideline. SIGN no. 83. Edinburgh: Scottish Intercollegiate Guidelines Network; 2005.

5. Rodd HD, Waterhouse PJ, Fuks AB, Fayle SA, Moffat MA, British Society of Paediatric Dentistry. Pulp therapy for primary molars. Int J Paediatr Dent 2006; 16 (suppl. 1): S15-S23.

6. Kunz R, Vist G, Oxman AD. Randomisation to protect against selection bias in healthcare trials. Cochrane Database Methodol Rev 2002; issue 4.

Evidence-Based Dentistry (2007) 8, 11-12. doi:10.1038/sj.ebd.6400464 